\title{
What Is Your Diagnosis?
}

In collaboration with the American College of Veterinary Radiology

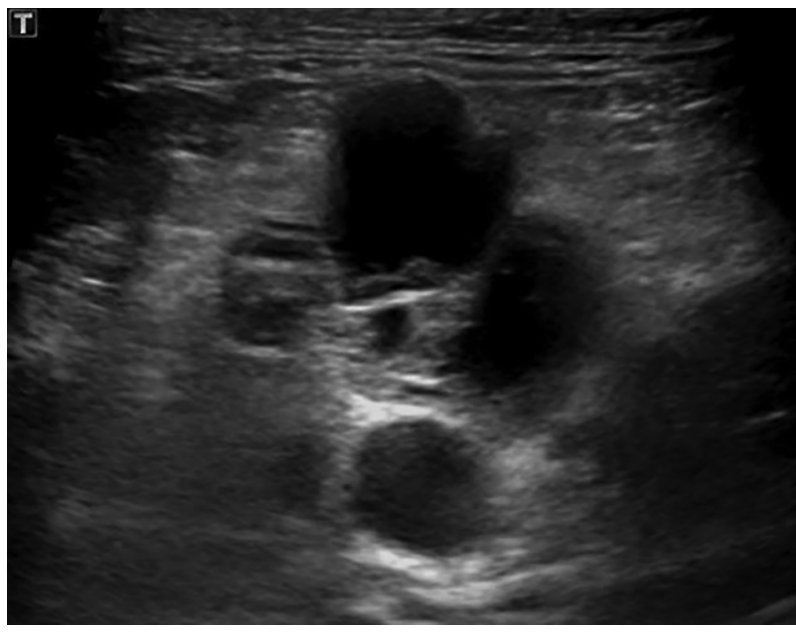

Figure I-Transverse abdominal ultrasonographic image obtained at the level of the pancreas of a 13-year-old 4.9-kg male castrated Egyptian Mau cat evaluated because of a 2-month history of inappetence, weight loss, persistent diarrhea, and lethargy. The cat's right side is toward the left of the image.

\section{History}

A 13-year-old 4.9-kg male castrated Egyptian Mau cat was presented because of a 2-month history of inappetence, weight loss, persistent diarrhea, and lethargy. The cat's previous medical history included the following: chronic kidney disease classified as International Renal Interest Society stage $1,{ }^{1}$ suspected idiopathic renal glucosuria, bilateral cranial cruciate ligament disease, osteoarthritis of multiple joints, idiopathic hypercalcemia, chronic bronchitis, suspected allergic dermatitis, and suspected

Keira Sztukowski, BA'; Timothy B. Kloer, DVM, MS'; Tawfik Aboellail, BVSc, MVSc, PhD²; Giovanni Tremolada, DVM, MS, PhD³; Lynn R. Griffin, DVM, MS ${ }^{*}$

'Department of Environmental and Radiological Health Sciences, Veterinary Teaching Hospital, College of Veterinary Medicine and Biomedical Sciences, Colorado State University, Fort Collins, CO ${ }^{2}$ Department of Microbiology, Immunology, and Pathology, Veterinary Teaching Hospital, College of Veterinary Medicine and Biomedical Sciences, Colorado State University, Fort Collins, CO

${ }^{3}$ Department of Clinical Sciences, Veterinary Teaching Hospital, College of Veterinary Medicine and Biomedical Sciences, Colorado State University, Fort Collins, CO

*Corresponding author: Dr. Griffin (lynn.griffin@colostate.edu)

https://doi.org/I0.2460/javma.19.12.0652 inflammatory bowel disease. The cat was receiving budesonide ( $1 \mathrm{mg}$, PO, q $48 \mathrm{~h}$ ) for treatment of suspected inflammatory bowel disease.

On presentation, the cat was bright, alert, and responsive but had a body condition score of 3/9 with substantial cachexia and moderate atrophy of all major muscle groups. Other abnormal findings on physical examination included a tense abdomen and a large, palpable, irregularly contoured mass that filled most of the abdominal cavity. This mass was not definitively attributable to any specific organ.

A CBC revealed mild microcytic regenerative anemia with a PCV of $25 \%$ (reference range, $32 \%$ to $47 \%$ ), mean corpuscular volume of $38 \mathrm{fl}$ (reference range, 40 to $52 \mathrm{fl}$ ), reticulocyte count of $148.2 \times 10^{3}$ cells $/ \mu \mathrm{L}$ (reference range, 0 to $50.0 \times 10^{3}$ cells $/ \mu \mathrm{L}$ ), mild neutrophilia $\left(16.4 \mathrm{X} 10^{3}\right.$ cells/ $\mu \mathrm{L}$; reference range, $2.0 \mathrm{X}$ $10^{3}$ to $12.0 \mathrm{X} 10^{3}$ cells $\left./ \mu \mathrm{L}\right)$, and lymphopenia $(0.7 \mathrm{X}$ $10^{3}$ cells $/ \mu \mathrm{L}$; reference range, $1.5 \times 10^{3}$ to $6.0 \times 10^{3}$ cells $/ \mu \mathrm{L})$. The cat was sedated for abdominal ultrasonography (Figure I).

Formulate differential diagnoses, then continue reading.

\section{Diagnostic Imaging Findings and Interpretation}

Abdominal ultrasonographic examination revealed a heterogeneous, poorly marginated mass in the region of the pancreas with multiple variablesized, anechoic to hypoechoic, thinly walled, complex cystic structures (Figure 2). The entirety of the pancreas was effaced by the mass, which extended to the caudal third of the abdomen and displaced intestines to the right. Some of the cystic structures contained a mild to moderate amount of echogenic debris and showed evidence of partial to complete septation. No internal vascularity of the cystic mass was noted on color flow Doppler interrogation (not shown), and no abdominal lymphadenopathy was evident.

The cat underwent general anesthesia for abdominal CT with an angiography, which revealed the pancreas to have been expanded by and associated with multiple large fluid-attenuating and soft tissueattenuating structures with thin septation and rims of contrast enhancement (Figure 3). The cystic mass extended from the caudal aspect of the liver to the level of L6, filled most of the cranial portion and left mid to caudal portions of abdomen, and surrounded 


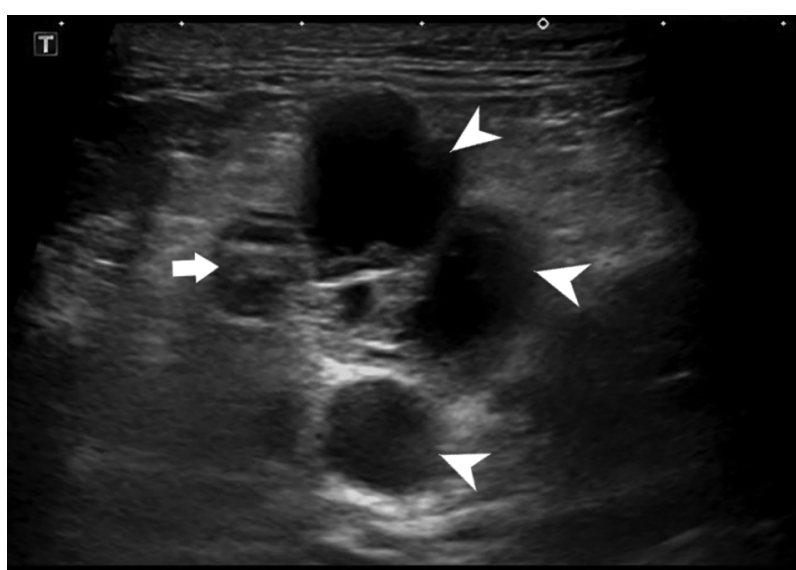

Figure 2-Same image as in Figure I. In the region of the pancreas and adjacent to the duodenum (arrow), there are large cystic structures (arrowheads) that are anechoic to hypoechoic and have thin walls and distal acoustic enhancement.

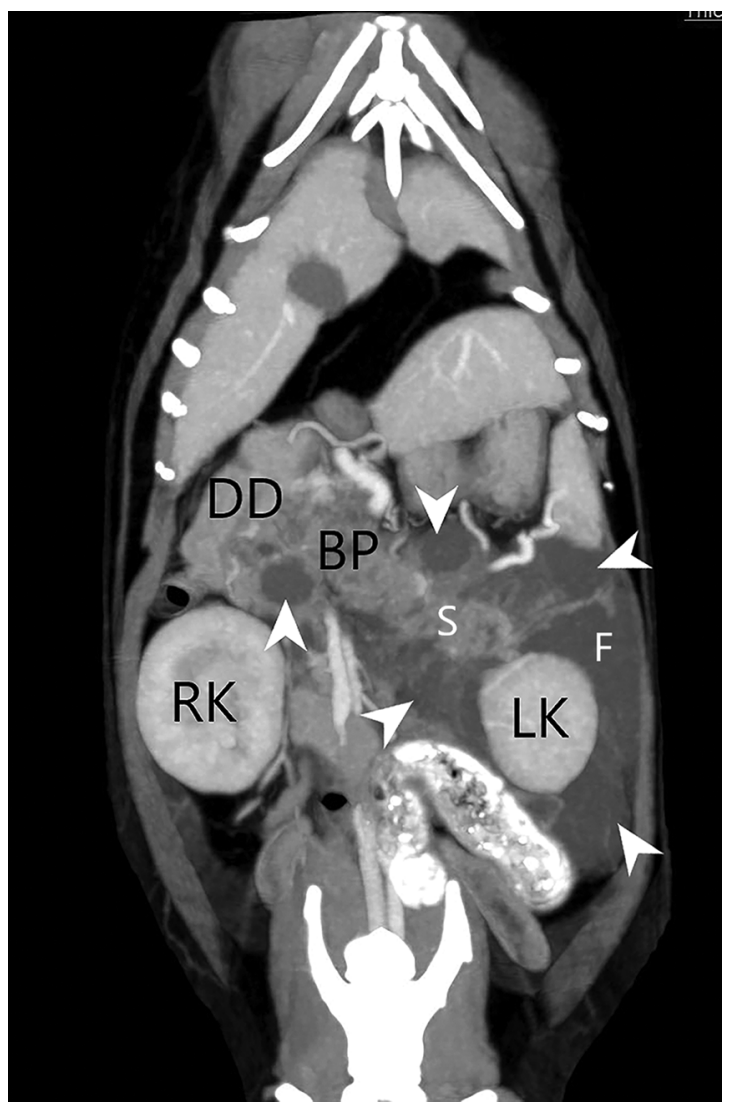

Figure 3-Venous phase dorsal plane maximal intensity projection CT angiographic image of the abdomen of the cat described in Figure I. The pancreas is expanded by and associated with multiple large fluid-attenuating and soft tissueattenuating structures (arrowheads) that occupy most of the cranial and left mid to caudal regions of the abdomen, extending from the caudal aspect of the liver to the level of L6. The cat's head and right side are toward the top and left side of the image, respectively. $\mathrm{BP}=$ Body of the pancreas. $\mathrm{DD}=$ Descending duodenum. $\mathrm{F}=$ Fluid-attenuating region of the abdominal mass. LK = Left kidney. RK = Right kidney. $S=$ Soft tissue-attenuating region of the abdominal mass. the cranial mesenteric and portal veins. Differential diagnoses included pancreatic pseudocysts, true cysts, polycystic pancreatic disease, and neoplasia, such as cystadenocarcinoma.

\section{Treatment and Outcome}

Cytology of an ultrasound-guided fine-needle aspirate sample of the cystic mass revealed cystic fluid, moderate histiocytic inflammation, exocrine pancreatic tissue, and evidence of chronic hemorrhage. Exploratory laparotomy was elected to obtain biopsy samples for histopathology and revealed a large cystic mass (approx $15 \times 17 \times 10 \mathrm{~cm}$ ) along with a newly formed brown layer of tissue covering the peritoneal surface, omentum, and spleen. Biopsy samples of the liver and omentum were obtained, and histologic examination revealed mild lymphoplasmacytic hepatitis and multinodular histiocytic omentitis with intrahistiocytic pigment granulomas, respectively. Because the cystic mass was friable and closely associated with the portal vein and descending duodenum, it was deemed not amenable to complete resection; however, a biopsy sample (approx $1 \mathrm{X} 1 \mathrm{X}$ $1 \mathrm{~cm})$ was obtained.

Histopathologic diagnosis of the mass was pancreatic acinar cell cystadenoma. Because resection was not a viable option, ongoing patient monitoring every 3 months with abdominal ultrasonography and bloodwork was recommended. Three months later, the patient was returned with abdominal effusion and suspected cyst rupture. Although the cat's clinical signs improved markedly upon drainage of the abdominal fluid and provision of supportive care, with no options for curative treatment and a high chance of repeated cyst rupture, the owners elected euthanasia for the cat.

\section{Comments}

Pancreatic acinar cell cystadenoma is a rare and poorly described tumor. ${ }^{2}$ To the author's knowledge, the present report was only the second case reported in cats. Other differential diagnoses for cystic lesions of the pancreas in cats include true cysts (either congenital or acquired retention cysts), pseudocysts, polycystic pancreatic disease, ductal and acinar adenoma, and cystadenocarcinoma. ${ }^{3}$ Due to prognostic implications, differentiating between cystic pancreatic lesions is of high importance. Pancreatic adenocarcinoma is an aggressive tumor with a high metastatic rate and poor prognosis in cats, having a mean survival time of 6 to 7 days without treatment and 165 days with surgical excision and chemotherapy. ${ }^{4}$ The prognosis of acinar cell cystadenoma in cats is unclear as there are no reports of long-term follow-up, but all cases reported in humans have been benign., ${ }^{5,6}$ The treatment of choice for large cystadenomas is complete excision, if possible. ${ }^{6}$ 
Imaging modalities must be combined with cytology and histopathology to accurately distinguish benign and malignant cystic pancreatic lesions and to recommend appropriate treatment or palliative options. Ultrasonographic and CT examinations were crucial in the initial diagnostic steps and decision to move forward with an exploratory laparotomy for the cat of the present report. The modalities provided information on the tumor's location, size, infiltration, metastasis, and relationship to peripheral tissues.

Ultrasonography alone can prove difficult to determine the organ of origin for extensive abdominal masses. For the cat of the present case, a mass originating from the liver could not be ruled out with ultrasonography due to the possibility of an eccentric pedunculated mass, the pedicle of which may have been missed. Computed tomography was especially useful in determining the organ of origin and the extent of the cystic lesions, which aided in surgical planning.

\section{References}

1. Munday JS, Lohr CV, Kiupel M. Tumors of the alimentary tract. In: Meuten DJ, ed. Tumors in Domestic Animals. 5th ed. John Wiley \& Sons Inc; 2017:499-601.

2. Linderman MJ, Brodsky EM, de Lorimier LP. Feline exocrine pancreatic carcinoma: a retrospective study of 34 cases. Vet Comp Oncol. 2013;11(3):208-218. doi:10.1111/j.14765829.2012.00320.x.

3. Klimstra DS, Hruban RH, Kloppel G, et al. Acinar cell neoplasms of the pancreas. In: Bosman FT, ed. WHO Classification of Tumors of the Digestive System. 4th ed. International Agency for Research on Cancer; 2010:314-318.

4. Wang G, Liang J, Qu F. Acinar cell cystadenoma of the pancreas: A retrospective analysis of ten-year experience from a single academic institution. Pancreatology. 2016;16(4): 625-631. doi:10.1016/j.pan.2016.03.020.

5. International Renal Interest Society Guidelines. 2019. Available at: www.iris-kidney.com/pdf/IRIS_Staging_of_CKD_ modified_2019.pdf. Accessed Oct 26, 2021.

6. Yoshimura H, Matsuda Y, Kawamoto Y, et al. Acinar cell cystadenoma of the pancreas in a cat. J Comp Pathol. 2013;149 (2-3):225-228. doi:10.1016/j.jcpa.2013.01.006. 